\title{
Differential Impact of Systemic Lymphadenectomy Upon the Survival of Patients with Type I vs Type II Endometrial Cancer: A Retrospective Observational Cohort Study
}

This article was published in the following Dove Press journal:

Cancer Management and Research

Jie $\mathrm{Xu}^{1,2}$

Can $\mathrm{Chen}^{3,4}$

Jing Xiong ${ }^{5}$

Hua Linghu ${ }^{1,2}$

'Department of Obstetrics and Gynecology, The First Affiliated Hospital of Chongqing Medical University, Chongqing 400016, People's Republic of China; ${ }^{2}$ The First Clinical College, Chongqing Medical University, Chongqing 4000 16, People's Republic of China; ${ }^{3}$ Department of Obstetrics and Gynecology, Chengdu Women \& Children's Central Hospital, Sichuan, 61009I, People's Republic of China; ${ }^{4}$ School of Medicine, University of Electronic Science and Technology of China, Chengdu 6II73I, People's Republic of China; ${ }^{5}$ Department of Obstetrics and Gynecology, Chongqing Health Center for Women and Children, Chongqing 40002I, People's Republic of China
Correspondence: Hua Linghu

Department of Obstetrics and Gynecology, The First Affiliated Hospital of Chongqing

Medical University, Chongqing 400016,

People's Republic of China

Tel +86-23-890I 1090

Fax+86-23-688II487

Email linghu_hua@yahoo.com
Purpose: To determine whether systemic lymphadenectomy exerts a similar effect on the survival of patients with either type I or type II endometrial cancer (EC).

Patients and Methods: In this retrospective study, 682 eligible patients diagnosed with EC were typed according to the pathological reports. The thoroughness of lymphadenectomy was evaluated by the lymph node number of which the cut-off value was determined by the receiver operator characteristic (ROC) curve and Youden index. The impact of thoroughness on the survival of both types was analyzed, respectively, by Kaplan Meier (K-M) method and further evaluated in subgroups with and without lymphatic metastasis. Independent prognostic factors of survival were selected by proportional hazard regression (Cox) model.

Results: The cut-off level of lymph node number was 20. The differential impact of the lymph node number removed on survival was noted when patients with different types were analyzed separately. Among type II EC, those with $>20$ lymph nodes removed presented better overall survival (OS) than those with $\leq 20$ ( $p=0.002$ ). The number of lymph nodes removed $>20$ was proved as an independent factor for improved OS in type II EC (HR $=0.329,95 \%$ CI: $0.123-$ $0.881, p=0.0027$ ). In the subgroup of type II with $>20$ lymph nodes resected, similar 5 -year OS rates were observed in those with or without identified positive node ( $90.9 \%$ vs $92.9 \%, p=0.965)$. Type I EC seemed unbeneficial from such a procedure.

Conclusion: Systemic lymphadenectomy could enhance the OS of type II EC other than type I.

Keywords: endometrial cancer, type, systemic lymphadenectomy, overall survival

\section{Introduction}

Endometrial cancer is one of the most common gynecologic cancers in developed countries $^{1}$ and of which the incidence has been increasing in China recently. ${ }^{2}$ Compared with type I EC, patients with type II EC usually present poorer survival because it is more aggressive and poorly differentiated. Surgical treatment is the optimal treatment for EC. Despite as an essential procedure of surgical staging of $\mathrm{EC}$, the impact of the lymphadenectomy on prognosis is still controversial. ${ }^{3}$ National Comprehensive Cancer Network (NCCN) guidelines in the United States and guidelines in Europe have included lymphadenectomy in surgical staging for EC. However, details about the thoroughness of dissection are still not specified. ${ }^{4}$ On the other hand, the prevalence of lymphadenectomy-associated comorbidities is generally reported and 
should not be ignored. ${ }^{5}$ Based on certain previous retrospective studies, the prognostic significance of lymphadenectomy was not observed in all EC, but in selective groups of cases such as EC with medium-risk and high-risk, high-grade EC and non-endometrioid EC. ${ }^{3,6,7}$ Accordingly, considering the differences between EC with two types, we conducted an observational cohort study to testify whether the thoroughness of lymphadenectomy would exert a differential impact on the survival of type I vs type II EC patients.

\section{Methods}

\section{Data Resources}

From May 2011 to May 2017, 724 patients diagnosed with EC were admitted to the First Affiliated Hospital of Chongqing Medical University in China. Patients were excluded who had already received staging surgery at other hospitals, refused any therapy at diagnosis, and were complicated with malignancies from other organs. Standardized staging surgery was performed in 688 patients. Incomplete data were found in 6 patients, who were excluded equally. Ultimately, 682 patients were enrolled. Forty patients lost during follow-up were included as censored values, and the missing rate was 5.8\% (Figure 1). Among patients enrolled, 519 of whom were type I EC and 163 were type II EC. Informed consent was obtained from all subjects. This study was conducted in accordance with Declaration of Helsinki. All experimental protocols were approved by the Ethics Committee of the First Affiliated Hospital of Chongqing Medical University.

Demographic information includes age, body mass index (BMI), menopause at diagnosis and post-operative adjuvant treatment. The staging surgeries, including extrafascial hysterectomy+bilateral adnexectomy+ pelvic lymphadenectomy \pm para-aortic lymphadenectomy (PALND), were performed by ten gynecologists with surgical experience of at least five years. All methods were carried out in accordance with relevant guidelines and regulations. Postoperative pathological report described myometrial invasion, lymphovascular space invasion, adnexal metastasis, histological type and grade, lymphatic metastasis, as well as number of dissected lymph nodes.

\section{Thoroughness of Lymphadenectomy}

The thoroughness of lymph node dissection was evaluated by the number of dissected lymph nodes, ${ }^{8}$ and the cut-off value is determined by the ROC curve and Youden index. The anatomic extent of standard lymphadenectomy for EC includes

\section{Patients with endometrial cancer from May 2011 to May 2017 in the First Affiliated Hospital of Chongqing Medical University $(n=724)$}

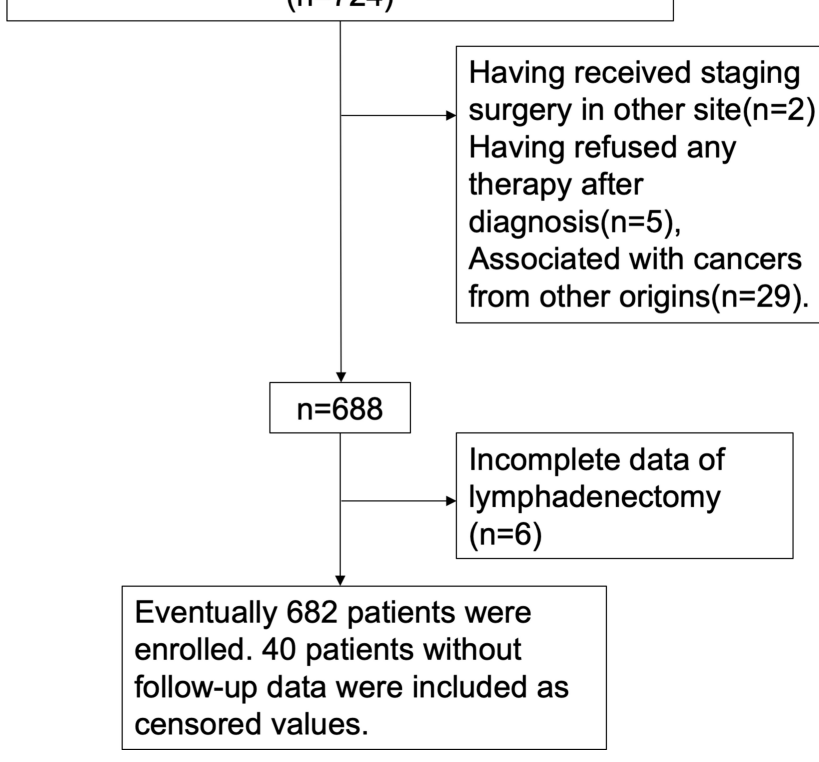

Figure I Consort flow diagram.

the pelvic and para-aortic region. The decision of PALND was made by the surgeons according to preoperative pathological reports and radiographic data as well as intraoperative findings.

\section{Type of Endometrial Cancer}

Type I EC is pathologically defined as grade $1 / 2$ endometrioid adenocarcinoma (or endometrioid adenocarcinoma with squamous differentiations) while Type II EC, the grade 3 endometrioid adenocarcinoma (or endometrioid adenocarcinoma with squamous differentiations) or highgrade non-endometrioid carcinoma (including endometrial serous carcinoma, clear cell carcinoma, squamous carcinoma, adenosquamous carcinoma, mixed carcinoma and undifferentiated carcinoma). ${ }^{9}$

\section{Statistical Analysis}

The categorical variables were evaluated by the Pearson chi-square test. Survival analyses were conducted by the K-M method. The Log rank test was used to estimate the differences in survival. The cut-off value of dissected nodes was determined together by the ROC curve and Youden index. Multivariate analyses were performed by the Cox model. $p<0.05$ was considered statistically significant in all tests in software SPSS of version 23.0. 


\section{Results}

\section{Demographic Characteristics}

Demographic characteristics of 682 patients with two types of EC are illustrated in Table 1. The median age of the patients is 52 (27-89), and the median BMI is 24.44 (16.35-42.24). Patients with two types have obvious differences in the International Federation of Gynecology and Obstetrics (FIGO) stage and tumor invasion indicators including myometrial invasion, tumor size, adnexal metastasis, and lymphovascular space invasion. More patients with type II EC were diagnosed at postmenopausal age, compared to those with type I $(70.6 \%$ vs $50.9 \%, p<$ $0.001)$; more received post-operative treatments $(89.6 \%$ vs $51.3 \%, p<0.001)$. No significant difference in surgical approach (laparotomy or laparoscopy) and obesity is observed. The rate of laparoscopy, respectively, is 55.8\% vs $61.8 \%(p=0.199)$ and the proportion of BMI $>24$ is $51.5 \%$ vs $54.9 \%(p=0.472)$ (Table 1$)$.

543 of these 682 patients had received pelvic lymphadenectomy, and 60 of whom had extra para-aortic lymphadenectomy. The number of total dissected nodes, negative nodes, and positive nodes were attained in the postoperative pathology report. The median number of total dissected nodes is $26(1-76)$, the median number of positive nodes dissected is $2(1-17)$, and the median number of positive nodes dissected is $26(0-76)$.

Table I Demographic Characteristic of Patients $(n=682)$

\begin{tabular}{|c|c|c|c|c|c|c|}
\hline Characteristics & Type I & $\%$ & Type II & $\%$ & Total & $p$-value \\
\hline Age $\leq 55$ y (\%) & & & & & & $<0.001$ \\
\hline Yes & 330 & 63.6 & 76 & 46.6 & 406 & \\
\hline No & 189 & 36.4 & 87 & 53.4 & 276 & \\
\hline Menopause at diagnosis (\%) & & & & & & $<0.001$ \\
\hline No & 255 & 49.1 & 48 & 29.4 & 303 & \\
\hline Yes & 264 & 50.9 & 115 & 70.6 & 379 & \\
\hline $\mathrm{BMI} \leq 24 \mathrm{~kg} / \mathrm{m}^{2}(\%)$ & & & & & & 0.472 \\
\hline Yes & 234 & 45.1 & 79 & 48.5 & 313 & \\
\hline No & 285 & 54.9 & 84 & 51.5 & 369 & \\
\hline Surgical approach (\%) & & & & & & 0.199 \\
\hline Laparotomy & 198 & 38.2 & 72 & 44.2 & 270 & \\
\hline Laparoscopy & 321 & 61.8 & 91 & 55.8 & 412 & \\
\hline Adjuvant treatment (\%) & & & & & & $<0.001$ \\
\hline Yes & 266 & 51.3 & 146 & 89.6 & 412 & \\
\hline No & 253 & 48.7 & 17 & 10.4 & 270 & \\
\hline FIGO stage (\%) & & & & & & $<0.001$ \\
\hline I/II & 489 & 94.2 & 102 & 62.6 & 591 & \\
\hline III/IV & 30 & 5.8 & 61 & 37.4 & 91 & \\
\hline Myometrial invasion (\%) & & & & & & $<0.001$ \\
\hline$\leq \mathrm{I} / 2$ & 433 & 83.4 & 93 & 57.1 & 526 & \\
\hline$>\mathrm{I} / 2$ & 86 & 16.6 & 70 & 42.9 & 156 & \\
\hline Size of tumor $\leq 2 \mathrm{~cm}$ & & & & & & $<0.001$ \\
\hline Yes & 249 & 48.0 & 46 & 28.2 & 295 & \\
\hline No & 270 & 52.0 & 117 & 71.8 & 387 & \\
\hline Adnexal metastasis (\%) & & & & & & $<0.001$ \\
\hline Negative & 506 & 97.5 & 138 & 84.7 & 644 & \\
\hline Positive & 13 & 2.5 & 25 & 15.3 & 38 & \\
\hline Lymphovascular space invasion (\%) & & & & & & $<0.001$ \\
\hline Negative & 503 & 96.9 & $|4|$ & 86.5 & 644 & \\
\hline Positive & 16 & 3.1 & 22 & 13.5 & 38 & \\
\hline
\end{tabular}




\section{Survival Analysis}

More than 20 (the cut-off value) lymph nodes are considered the systemic lymphadenectomy. The mean OS of type I and type II cohort are 72.7 months $(95 \%$ CI: 71.8-73.7) and 53.6 months (95\% CI: 49.5-57.7), the K-M method was conducted to make the comparison of OS between $\leq 20$ and $>20$ dissected nodes in type I EC (Figure 2A). More than 20 dissected nodes could not be proved to increase the OS of type I EC $(p=0.784)$.

Type II EC has three times more chance of lymphatic metastasis compared with type I. ${ }^{10} \mathrm{~K}-\mathrm{M}$ method was also conducted to make the comparison of OS between $\leq 20$ and $>20$ dissected nodes in type II EC (Figure 2B), and the 5-year OS rates were $55.0 \%$ and $89.2 \%(p=0.002)$, suggesting that the OS of patients with $>20$ dissected nodes was statistically longer than that of patients with $\leq 20$ lymph nodes dissected.
Subgroup analysis: Further analyses were conducted to compare the OS of type II EC with $\leq 20$ or $>20$ dissected nodes in cohorts with and without lymphatic metastasis. For those with $\leq 20$ nodes dissected, the OS of positive node group was significantly worse, $p=0.014$ (Figure 3A). For those with $>20$ dissected nodes, despite with lymphatic metastasis, a similar OS was observed between the two cohorts $(p=0.965)$. (Figure 3B).

To verify whether or not the thoroughness of lymphadenectomy is an independent factor for OS of type II EC, multivariate analysis was conducted. Cox model enrolled factors selected by K-M analyses and those that were considered to have contributions to survival clinically. The results indicated that thoroughness of lymphadenectomy, the surgical approach, menopause or pre-menopause at diagnosis, myometrial invasion and the FIGO stage were independent prognostic factors affecting OS of type II EC. Early stage, laparoscopy, >20 dissected nodes,
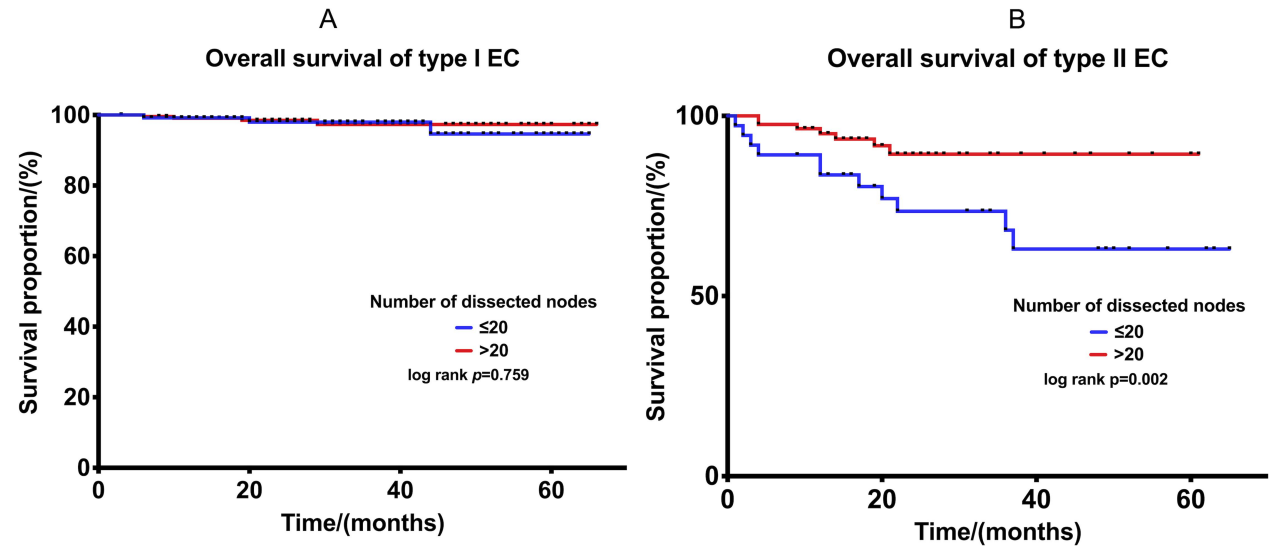

Figure 2 (A) Comparison of OS in type I EC between $\leq 20$ and $>20$ lymph nodes dissected. (B) Comparison of OS in type II EC between $\leq 20$ and $>20$ lymph nodes dissected.

A

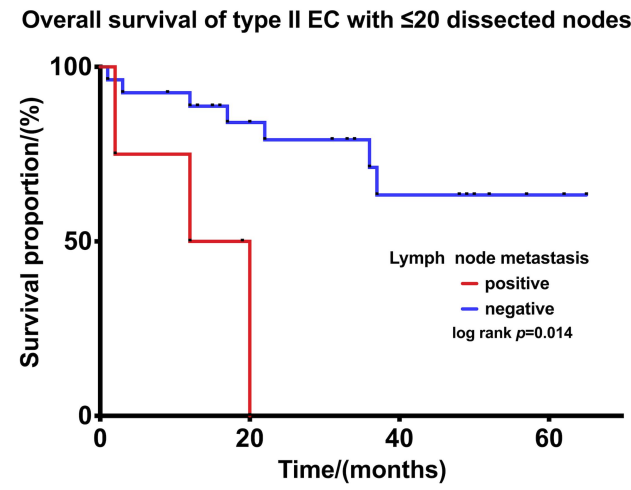

B

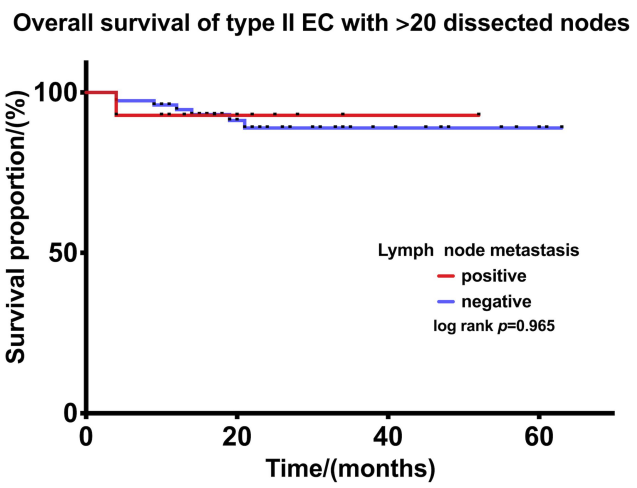

Figure 3 (A) Comparison of OS in patients with and without lymphatic metastasis in type II EC with $\leq 20$ lymph nodes dissected. (B) Comparison of OS in patients with and without lymphatic metastasis in type II EC with $>20$ lymph nodes dissected. 
Table 2 Cox-Regression Analysis in Type-II EC Patients ( $\mathrm{n}=$ 163)

\begin{tabular}{|c|c|c|}
\hline \multirow[t]{2}{*}{ Variables } & \multicolumn{2}{|l|}{ Overall Survival } \\
\hline & HR (95\% Cl) & P-value \\
\hline Multivariate analysis & & \\
\hline $\begin{array}{l}2016 \text { FIGO stage } \\
\text { I/II } \\
\text { III/IV }\end{array}$ & $\begin{array}{l}\text { I } \\
6.718(2.882-15.661)\end{array}$ & $<0.001$ \\
\hline $\begin{array}{l}\text { Myometrial invasion } \\
<1 / 2 \\
>I / 2\end{array}$ & $\begin{array}{l}1 \\
2.892(1.210-6.911)\end{array}$ & 0.017 \\
\hline $\begin{array}{l}\text { Surgical approach } \\
\text { Laparotomy } \\
\text { Laparoscopy }\end{array}$ & $\begin{array}{l}\text { I } \\
0.4 I I(0.172-0.98 I)\end{array}$ & 0.045 \\
\hline $\begin{array}{l}\text { No. total nodes obtained } \\
\quad \leq 20 \\
>20\end{array}$ & $\begin{array}{l}\text { I } \\
0.366(0.166-0.810)\end{array}$ & 0.013 \\
\hline $\begin{array}{l}\text { Menopause at diagnosis } \\
\text { No } \\
\text { Yes }\end{array}$ & $\begin{array}{l}\text { I } \\
3.055(1.023-9.117)\end{array}$ & 0.045 \\
\hline
\end{tabular}

pre-menopause and myometrial invasion $<1 / 2$ increases the OS rate of patients with type II EC (Table 2).

\section{Discussion}

This retrospective cohort study enrolled patients with EC of total 682. The different impact of thorough lymphadenectomy upon OS in type I and type II EC has been observed. Type II EC with $>20$ dissected nodes presented better OS compared with $\leq 20$, and systemic lymphadenectomy was further indicated as an independent factor affecting OS of type II EC. Besides, the subgroup with positive node benefits from systemic lymphadenectomy, reaching a similar OS of those without node metastasis.

The thoroughness of lymphadenectomy does not improve the OS of type I EC since its prognosis is already satisfying, even in subgroup of advanced stage (Figure S1, Table S1). Type II EC benefits from thorough lymph node dissection, possibly due to its greater invasiveness of cancer cells. Removement of lymph nodes as many as possible can theoretically reduce more cancer cells, especially micro-metastasis, which could further explain the results of subgroup analysis. Despite lymphatic metastasis, more than 20 dissected nodes are beneficial for reducing the risk of death in type II EC patients.
Lymphadenectomy has been recommended by the American College of Obstetricians and Gynecologists (ACOG) in surgical staging for EC patients since 2005. ${ }^{11}$ While the survival advantage of thorough lymph node dissection is still being questioned, the long-term complications have always been a problem to neglect. Systemic lymphadenectomy commonly leads to lymphedema in the lower extremities, especially during dissections in certain regions such as the circumflex iliac spinal lymph node. ${ }^{12}$ Since there is no optimal treatment for lower extremity lymphedema at present, lifetime care is needed in some severe cases. ${ }^{4,5}$ Recently, whether it is necessary to perform thorough lymphadenectomy for all EC patients has been controversial. Initially, Pierluigi et al found that systemic lymph node dissection does not increase OS and Progression-free survival for early endometrial cancer. ${ }^{13}$ But some other studies have found that in early-stage EC with moderate to high-risk factors for recurrence and pathological grades of $\mathrm{G} 3$, removal of $>11$ lymph nodes was associated with a better prognosis. ${ }^{14,15}$ And a retrospective study found that $>25$ pelvic and paraaortic lymph node dissection for high-grade EC could be an independent factor in reducing recurrent rates and benefiting long-term survival and disease-free survival. ${ }^{3}$ Another multicenter retrospective cohort study demonstrated that removal of as many lymph nodes as possible for moderately high-risk stage IIb-IIIc2 endometrioid carcinoma ensures an accurate staging and improves the prognosis. ${ }^{6}$ In some way, more clinicians seem to reach a consensus that systemic lymphadenectomy is not ideal for all EC. It reminds us of selectively performing systemic lymphadenectomy in a certain group of patients. With the premise of not worsening prognosis, complications brought by systemic lymphadenectomy could be avoided as far as possible. Except for the stage and the risk factor of recurrence and metastasis, the type should be also taken into consideration.

The 5-year OS of type I EC in this study is up to $98.4 \%$, which was not ameliorated by thorough lymphadenectomy. Besides no survival benefits have been observed, the rates of swelling and numbness caused by lymphadenectomy are up to $27 \%$ and $11.7 \%$. Therefore, for type I EC, thorough lymphadenectomy would not be recommended according to our results.

The sentinel node (SLN) biopsy and dissection have come up as an alternative staging technique. Its ability of detection in pelvic lymphatic metastasis has been verified in early-stage $\mathrm{EC},{ }^{16}$ but the detection rate of the para- 
aortic lymph node is still low. ${ }^{17-19}$ For another, the isolated para-aortic lymph node metastasis in high-risk EC was reported to be $16 \%,{ }^{16}$ which should not be ignored. Interestingly, Tae et al have discovered when there are $>30$ dissected nodes, the rate of isolated para-aortic lymph node metastasis is $0 \%{ }^{6}$ suggesting the possibility of such metastasis could be diminished by systemic lymph node resection. The pelvic lymph node metastasis of type II is three times more than type $\mathrm{I} \mathrm{EC}^{10}$ and Marcos et al observed all three patients in their study with falsenegative SLN had type II EC. ${ }^{20}$ The thorough lymphadenectomy seems not avoidable for type II EC with its detection ability and survival benefit. Consequently, the pre-operative diagnosis of type is of great importance. The concordance rates of the pre-operative and final pathological report are reported to be varied between $56 \%-81 \%$. $7.2 \%$ had downgrade discordance, and the most common type of downgrade was seen in type I EC. ${ }^{21}$ Based on the low possibility of downgrade post-operatively, thorough lymphadenectomy could be suggested for the curettagediagnosed type II EC. Other strategies such as combined diagnosis might increase the accuracy of curettage.

A similar study has noticed the impact of systemic lymphadenectomy on non-endometrioid EC. The study from American Sriram Venigalla et al have reported the thoroughness of lymphadenectomy is an independent prognostic factor for risk of death in patients with nonendometrioid EC, and the cut-off value was $15 .^{7}$ In this study, the G3 endometrioid endometrial cancer (EEC) is included together with non-endometrioid EC. The type of EC was firstly proposed in 1983 by Bohkman, ${ }^{22}$ since then the classification of G3 EEC has always been controversial, as it has both features of type I and type II EC. ${ }^{23-25}$ In this study, G3 EEC was classified as type II EC as most researchers do. ${ }^{26-28}$ Although guidelines of NCCN and SGO have recently recommended the inclusion of SLN mapping for EC patients, the European Society of Gynecological Oncology (ESGO) has been skeptical about it. ${ }^{4}$ The SLN biopsy is only recommended as a proper alternative for early-stage and intermediate-high risk EC. And the lymphadenectomy is recommended for EC with high risk of recurrence, G3 EEC and nonendometrioid $\mathrm{EC}^{29}$ which is precisely consistent with type II in our study.

The dissection of suspicious enlarged nodes is suggested by the NCCN guideline. Pre-operative radiography is an alternative technique to help presuppose lymph node metastasis. The high sensitivity and specificity in detecting lymphatic metastasis of Magnetic Resonance Imaging (MRI) have been reported in a retrospective study, ${ }^{30}$ and its relatively higher accuracy rate was further verified by Koplay et al compared with B-mode and Computed Tomography (CT) scan, ${ }^{31}$ In this study, 533 patients underwent pelvic and abdominal CT or MRI before surgery. A total of 32 cases were positive for lymphatic metastasis, and 28 of which underwent preoperative radiography. In 17 of the 28 patients (Including 3 type I EC and 14 type II EC), pre-operative imaging indicates node disease. Eleven cases of lymph node metastasis were not detected by MRI/CT (false-negative rate was $2.1 \%$ ) and in 14 cases pre-operative lymphatic metastasis in MRI/CT was not further supported by pathology after surgery (false-positive rate was $2.6 \%$ ). The imaging performance in the early stages of cancer may not be typical, and the accuracy of the pre-operative assessment of lymphatic metastasis may depend on the resolution of imaging system and experience of radiographers as well. Meanwhile, it requires a gynecologic oncologist to enhance the ability to read imaging for ideal preoperative evaluation.

Laparoscopy was found to improve the OS of type II EC compared with open laparotomy in this study. Although the minimally invasive approach has been proved with no significant survival benefits for $\mathrm{OS},{ }^{32}$ for type II EC with a high possibility of metastasis, a bright surgical field of view could help perform a thorough lymphadenectomy.

One of the strengths of this study is the comparison of the survival benefits of thorough lymphadenectomy between two types of EC, providing practical evidence for clinical work. And the other is that the unity of staging surgery from a single center reduces confounding factors. One of the limitations lies in its retrospective nature. Second, the sample size was not enough in the subgroup analysis, resulting in a less strong conclusion.

\section{Conclusions}

For type II EC, thorough lymphadenectomy could improve the OS, and more than 20 lymph nodes dissected is an independent prognostic factor. For clinically early-stage EC, pre-operative pathological diagnosis, type and lymphatic metastasis have guiding value for surgery extent and range. Selectively performing lymphadenectomy could be recommended for pre-operatively and intraoperatively suspected type I EC, which was demonstrated not to improve the prognosis. In contrast, tendentious systemic lymphadenectomy could be performed for type II EC 
despite lymphatic metastasis. Related prospective research would further support our results. Preoperative diagnosis strategy of EC type by radiography and curettage could be further investigated to guide the staging surgery plan.

\section{Abbreviations}

EC, endometrial cancer; ROC, receiver operator characteristic; K-M, Kaplan-Meier; Cox, proportional hazard regression; OS, overall survival; NCCN, National Comprehensive Cancer Network; BMI, body mass index; PALND, paraaortic lymphadenectomy; FIGO, The International Federation of Gynecology and Obstetrics; ACOG, American College of Obstetricians and Gynecologists; SLN, sentinel node; EEC, endometrioid endometrial cancer; SGO, Society of Gynecologic Oncology; ESGO, European Society of Gynaecological Oncology; MRI, magnetic resonance imaging; CT, computed tomography.

\section{Data Sharing Statement}

The datasets generated during the current study are not publicly available but are available from the corresponding author on reasonable request.

\section{Acknowledgments}

I would like to show my deepest gratitude to Professor Yi Jing and Professor Qing Zeng (School of Public Health and Management, Chongqing Medical University) for all the help with statistical analysis.

\section{Disclosure}

The authors report no conflicts of interest in this work.

\section{References}

1. Siegel RL, Miller KD, Jemal A. Cancer statistics, 2019. CA Cancer J Clin. 2019;69(1):7-34. doi:10.3322/caac.21551

2. Lei J. The advancement of endometrial cancer. 2015

3. Papathemelis T, Scharl S, Kronberger K, et al. Survival benefit of pelvic and paraaortic lymphadenectomy in high-grade endometrial carcinoma: a retrospective population-based cohort analysis. $J$ Cancer Res Clin Oncol. 2017;143(12):2555-2562. doi:10.1007/ s00432-017-2508-1

4. Colombo N, Creutzberg C, Amant F, et al. ESMO-ESGO-ESTRO Consensus Conference on Endometrial Cancer: diagnosis, treatment and follow-up. Int J Gynecol Cancer. 2016;26(1):2-30. doi:10.1097/ IGC.0000000000000609

5. Biglia N, Zanfagnin V, Daniele A, Robba E, Bounous VE. Lower body lymphedema in patients with gynecologic cancer. Anticancer Res. 2017;37(8):4005-4015.

6. Kim TH, Kim HS, Kim T-J, et al. Survival impact based on the thoroughness of pelvic lymphadenectomy in intermediate- or high-risk groups of endometrioid-type endometrial cancer: a multi-center retrospective cohort analysis. Gynecol Oncol. 2016;141 (3):440-446. doi:10.1016/j.ygyno.2016.03.031
7. Venigalla S, Chowdhry AK, Shalowitz DI. Survival implications of staging lymphadenectomy for non-endometrioid endometrial cancers. Gynecol Oncol. 2018;149(3):531-538.

8. Todo Y, Kato H, Kaneuchi M, Watari H, Takeda M, Sakuragi N. Survival effect of para-aortic lymphadenectomy in endometrial cancer (SEPAL study): a retrospective cohort analysis. Lancet. 2010;375 (9721):1165-1172. doi:10.1016/S0140-6736(09)62002-X

9. Setiawan VW, Yang HP, Pike MC, et al. Type I and II endometrial cancers: have they different risk factors? J Clin Oncol. 2013;31 (20):2607-2618.

10. Suarez AA, Felix AS, Cohn DE. Bokhman Redux: endometrial cancer "types" in the 21st century. Gynecol Oncol. 2017;144 (2):243-249. doi:10.1016/j.ygyno.2016.12.010

11. Liu JH, Greer BE, Curtin J. ACOG Practice Bulletin\# 65: management of endometrial cancer. Obstet Gynecol. 2006;107(4):pp.952-953. doi:10.1097/01.AOG.0000209465.55688.30

12. Lindqvist E, Wedin M, Fredrikson M, Kjolhede P. Lymphedema after treatment for endometrial cancer - a review of prevalence and risk factors. Eur J Obstet Gynecol Reprod Biol. 2017;211:112-121. doi:10.1016/j.ejogrb.2017.02.021

13. Panici PB, Basile S, Maneschi F, et al. Systematic Pelvic lymphadenectomy vs no lymphadenectomy in early-stage endometrial carcinoma: randomized clinical trial. JNCI J National Cancer Inst. 2008;100(23):1707-1716. doi:10.1093/jnci/djn397

14. Li MY, Hu XX, Zhong JH, Chen LL, Lin YX. Therapeutic role of systematic lymphadenectomy in early-stage endometrial cancer: a systematic review. Oncol Lett. 2016;11(6):3849-3857. doi:10.3892/ol.2016.4467

15. Cusano E, Myers V, Samant R, et al. Prognostic significance of lymphovascular space invasion in the absence of lymph node metastases in early-stage endometrial cancer. Int $J$ Gynecol Cancer. 2018;28(5):890-894. doi:10.1097/IGC.0000000000001229

16. Togami S, Kawamura T, Fukuda M, Yanazume S, Kamio M, Kobayashi H. Prospective study of sentinel lymph node mapping for endometrial cancer. Int $J$ Gynecol Obstetrics. 2018;143 (3):313-318. doi:10.1002/ijgo.12651

17. Touhami O, Gregoire J, Renaud MC, Sebastianelli A, Plante M. Performance of sentinel lymph node (SLN) mapping in high-risk endometrial cancer. Gynecol Oncol. 2017;147(3):549-553. doi:10.1016/j.ygyno.2017.09.014

18. Soliman PT, Westin SN, Dioun S, et al. A prospective validation study of sentinel lymph node mapping for high-risk endometrial cancer. Gynecol Oncol. 2017;146(2):234-239. doi:10.1016/j. ygyno.2017.05.016

19. Rossi EC, Kowalski LD, Scalici J, et al. A comparison of sentinel lymph node biopsy to lymphadenectomy for endometrial cancer staging (FIRES trial): a multicentre, prospective, cohort study. Lancet Oncol. 2017;18(3):384-392. doi:10.1016/S1470-2045(17)30068-2

20. Ballester M, Dubernard G, Lécuru F, et al. Detection rate and diagnostic accuracy of sentinel-node biopsy in early stage endometrial cancer: a prospective multicentre study (SENTI-ENDO). Lancet Oncol. 2011;12(5):469-476. doi:10.1016/S1470-2045(11)70070-5

21. Shiozaki T, Miwa M, Sakuma T, et al. Correlation between pre-operative and final histological diagnosis on endometrial cancer. Int $J$ Gynecol Cancer. 2019;29(5):886-889. doi:10.1136/ijgc-2018-000041

22. Bokhman JV. Two pathogenetic types of endometrial carcinoma. Gynecol Oncol1983;151(1). doi:10.1016/0090-8258(83)90111-7

23. Di Cello A, Rania E, Zuccalà V, et al. Failure to recognize preoperatively high-risk endometrial carcinoma is associated with a poor outcome. Eur $J$ Obstetrics Gynecol Reproduct Biol. 2015;194:153-160. doi:10.1016/j.ejogrb.2015.09.004

24. Murali R, Delair DF, Bean SM, Abu-Rustum NR, Soslow RA. Evolving roles of histologic evaluation and molecular/genomic profiling in the management of endometrial cancer. $J$ National Comprehensive Cancer Network. 2018;16(2):201-209. doi:10.6004/ jnccn.2017.7066 
25. Ercelep O, Gumus M. Comparison of clinicopathologic and survival characteristics of high grade endometrial cancers; single center experience. Curr Probl Cancer. 2019;43(2):160-166. doi:10.1016/j. currproblcancer.2018.07.004

26. Voss MA, Ganesan R, Ludeman L, et al. Should grade 3 endometrioid endometrial carcinoma be considered a type 2 cancer a clinical and pathological evaluation. Gynecol Oncol. 2012;124 (1):15-20. doi:10.1016/j.ygyno.2011.07.030

27. Altman AD, Ferguson SE, Atenafu EG, et al. Canadian High Risk Endometrial Cancer (CHREC) consortium: analyzing the clinical behavior of high risk endometrial cancers. Gynecol Oncol. 2015;139(2):268-274. doi:10.1016/j.ygyno.2015.09.001

28. Milgrom SA, Kollmeier MA, Abu-Rustum NR, O’Cearbhaill RE, Barakat RR, Alektiar KM. Quantifying the risk of recurrence and death in stage III (FIGO 2009) endometrial cancer. Gynecol Oncol. 2014;134(2):297-301. doi:10.1016/j.ygyno.2014.05.013

29. Body N, Gregoire J, Renaud MC, Sebastianelli A, Grondin K, Plante M. Tips and tricks to improve sentinel lymph node mapping with Indocyanin green in endometrial cancer. Gynecol Oncol. 2018;150(2):267-273. doi:10.1016/j.ygyno.2018.06.001
30. Koplay M, Dogan NU, Erdogan H, et al. Diagnostic efficacy of diffusion-weighted MRI for pre-operative assessment of myometrial and cervical invasion and pelvic lymph node metastasis in endometrial carcinoma. J Med Imaging Radiat Oncol. 2014;58(5):538-546. doi:10.1111/1754-9485.12209

31. Lu R, Guixia S. Research into the value of B-mode ultrasound, CT and MRI examinations in the diagnosis of preoperative myometrial infiltration of endometrial cancer and lymph node metastasis. Open Med. 2015;10(1):388-393.

32. Deura I, Shimada M, Azuma Y, et al. Comparison of laparoscopic surgery and conventional laparotomy for surgical staging of patients with presumed low-risk endometrial cancer: the current state of Japan. Taiwan J Obstet Gynecol. 2019;58(1):99-104. doi:10.1016/j. tjog.2018.11.019

\section{Publish your work in this journal}

Cancer Management and Research is an international, peer-reviewed open access journal focusing on cancer research and the optimal use of preventative and integrated treatment interventions to achieve improved outcomes, enhanced survival and quality of life for the cancer patient.
The manuscript management system is completely online and includes a very quick and fair peer-review system, which is all easy to use. Visit http://www.dovepress.com/testimonials.php to read real quotes from published authors. 